\title{
Identification of a metabolism-related gene expression prognostic model in endometrial carcinoma patients
}

\author{
Pinping Jiang ${ }^{\dagger}$, Wei Sun $^{\dagger}$, Ningmei Shen ${ }^{\dagger}$, Xiaohao Huang $^{*}$ and Shilong Fu ${ }^{*}$ (I)
}

\begin{abstract}
Background: Metabolic abnormalities have recently been widely studied in various cancer types. This study aims to explore the expression profiles of metabolism-related genes (MRGs) in endometrial cancer (EC).

Methods: We analyzed the expression of MRGs using The Cancer Genome Atlas (TCGA) data to screen differentially expressed MRGs (DE-MRGs) significantly correlated with EC patient prognosis. Functional pathway enrichment analysis of the DE-MRGs was performed. LASSO and Cox regression analyses were performed to select MRGs closely related to EC patient outcomes. A prognostic signature was developed, and the efficacy was validated in part of and the entire TCGA EC cohort. Moreover, we developed a comprehensive nomogram including the risk model and clinical features to predict EC patients' survival probability.

Results: Forty-seven DE-MRGs were significantly correlated with EC patient prognosis. Functional enrichment analysis showed that these MRGs were highly enriched in amino acid, glycolysis, and glycerophospholipid metabolism. Nine MRGs were found to be closely related to EC patient outcomes: CYP4F3, CEL, GPAT3, LYPLA2, HNMT, PHGDH, CKM, UCK2 and ACACB. Based on these nine DE-MRGs, we developed a prognostic signature, and its efficacy in part of and the entire TCGA EC cohort was validated. The nine-MRG signature was independent of other clinical features, and could effectively distinguish high- and low-risk EC patients and predict patient OS. The nomogram showed excellent consistency between the predictions and actual survival observations.
\end{abstract}

Conclusions: The MRG prognostic model and the comprehensive nomogram could guide precise outcome prediction and rational therapy selection in clinical practice.

Keywords: Metabolism, TCGA, Endometrial carcinoma, Prognostic model, Nomogram

\section{Background}

Endometrial carcinoma (EC), one of the most common female reproductive malignancies, caused nearly 90,000 deaths worldwide each year [1]. Women with metabolic disorders, including obesity and diabetes, have a markedly increased risk of developing endometrial cancer. While early-stage endometrial cancer has a favorable

\footnotetext{
*Correspondence: xiaohao512006@126.com; docfusl@163.com †Pinping Jiang, Wei Sun and Ningmei Shen contributed equally to this work. Department of Gynecology, The First Affiliated Hospital of Nanjing Medical University, Nanjing 210029, Jiangsu Province, China
}

prognosis, nearly $30 \%$ of patients are still diagnosed at a late stage, and over $80 \%$ of these individuals die in 5 years [2]. In addition, several EC patients present a high risk of cancer progression or recurrence with insensitivity to chemotherapy, which indicates poor outcomes [3]. Therefore, it is imperative to emphasize the molecular changes that occur during endometrial cancer progression and develop novel predictive biomarkers to accurately estimate patient outcomes.

Since fundamental metabolic differences between cancer and adjacent normal cells were first uncovered,

C C The Author(s). 2020 Open Access This article is licensed under a Creative Commons Attribution 4.0 International License, which permits use, sharing, adaptation, distribution and reproduction in any medium or format, as long as you give appropriate credit to the original author(s) and the source, provide a link to the Creative Commons licence, and indicate if changes were made. The images or other third party material in this article are included in the article's Creative Commons licence, unless indicated otherwise in a credit line to the material. If material is not included in the article's Creative Commons licence and your intended use is not permitted by statutory regulation or exceeds the permitted use, you will need to obtain permission directly from the copyright holder. To view a copy of this licence, visit http://creativecommons.org/licenses/by/4.0/ The Creative Commons Public Domain Dedication waiver (http://creativecommons.org/publicdomain/zero/1.0/) applies to the data made available in this article, unless otherwise stated in a credit line to the data. 
metabolic reprogramming has increasingly become a hot topic in cancer biology [4]. The metabolic phenotype of cancer cells is heterogeneous in various cancer types; for example, while several malignant tumors mainly rely on glycolysis, others present a metabolic phenotype mediated by oxidative phosphorylation $[5,6]$. Overall, through reprogramming tumor microenvironments, catabolic and anabolic metabolism is essential for cancer cells to sustain energy supply and biomass synthesis [7-9]. While the underlying processes and molecular alterations of metabolic programming in various cancers have been well elucidated, the expression patterns of metabolism-related genes in endometrial cancer are still unclear.

In this study, we focused on the metabolism-related gene expression alterations of The Cancer Genome Atlas (TCGA) EC patients and obtained prognostic dysregulated MRGs. In addition, we established and validated a multiple-MRG-combined expression signature for predicting EC patient outcomes. Moreover, we integrated the clinical features of patients and the MRG model to establish a novel nomogram model that could guide comprehensive EC therapeutic strategies.

\section{Methods}

Integration of gene expression profiles and clinical information

We downloaded the mRNA expression profiles of EC patients (in the FPKM format) from The Cancer Genome Atlas (TCGA) database (https://tcga-data.nci.nih. gov/tcga/), which contains a total of 541 cases. The cBioPortal for Cancer Genomics (http://cbioportal.org) enables researchers to explore, visualize, and analyze multi-dimensional cancer genomics data and clinical information. The corresponding clinical data of $\mathrm{EC}$ was retrieved from the cBioPortal [10].

\section{Extraction of metabolism-related genes from the TCGA database}

Genes enriched in metabolic pathways in the KEGG database were utilized in this study as metabolic genes (Supplementary Table 1) [11]. The mRNA expression of metabolic genes in the TCGA database was extracted.

\section{Identification of prognosis-associated differentially expressed metabolism-related genes (DE-MRGs)}

With the cut-off criteria set as $|\operatorname{logFC}|>1$ and $P$-value < 0.05 , we screened the DE-MRGs via the "limma" R package [12]. Then, univariate Cox regression analysis was performed to identify prognosis-associated DE-MRGs. Hazard ratio $(\mathrm{HR})<1$ indicates better overall survival (OS) outcomes while HR $>1$ indicates worse OS outcomes. Genes with $P<0.05$ were regarded as prognosisassociated metabolic genes. The expression levels of the prognosis-associated DE-MRGs in each patient and between cancerous and normal samples were displayed via the "pheatmap" and "ggplot" R packages, respectively.

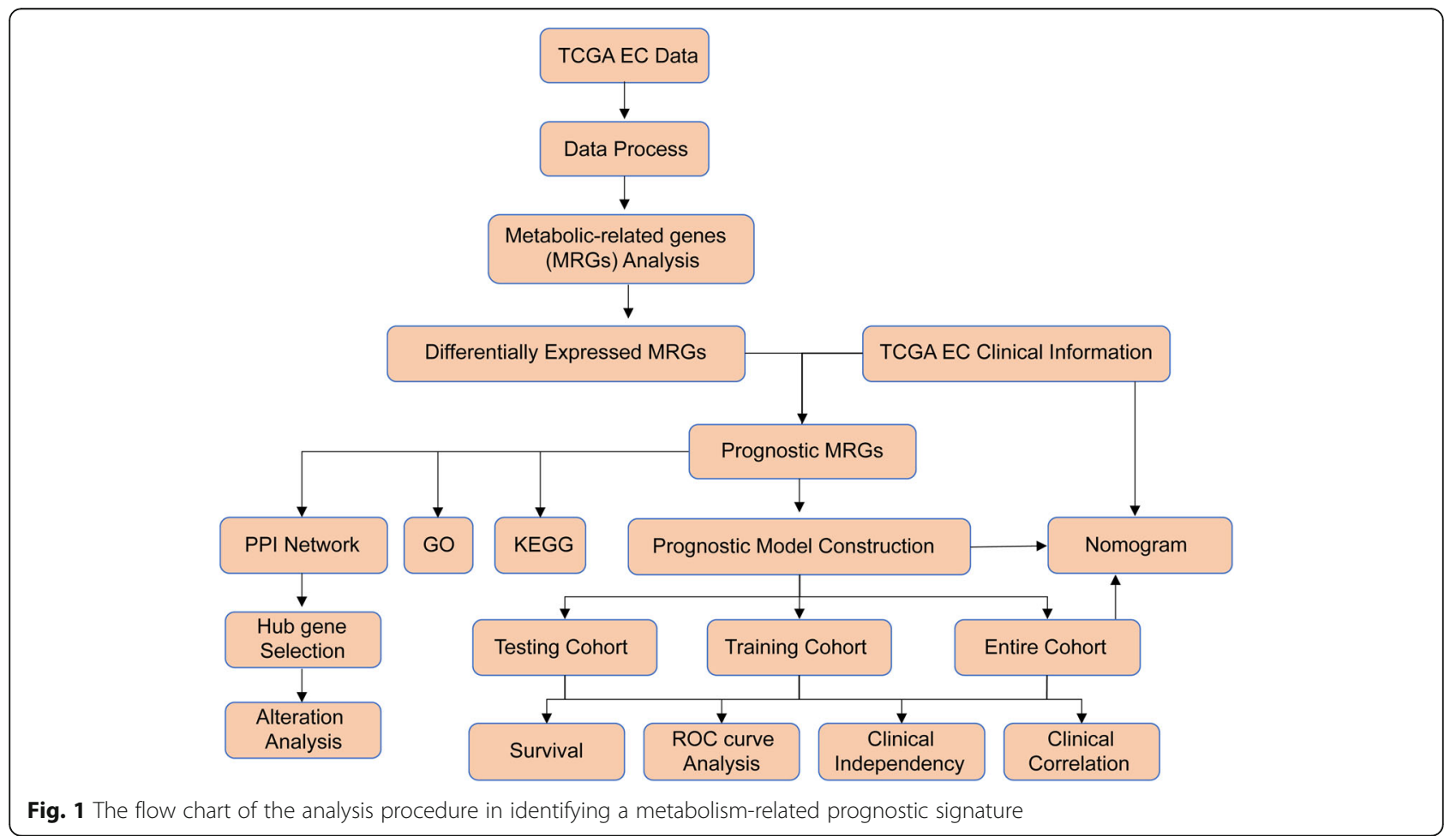




\section{Functional enrichment analysis of the prognosis- associated DE-MRGs}

Gene ontology (GO) [13] and Kyoto Encyclopedia of Genes and Genomes (KEGG) [11] pathway enrichment analyses were performed to explore the biological functions of the prognosis-related DE-MRGs via the "clusterProfiler" $\mathrm{R}$ package. Adjusted $P$-value $<0.05$ was set as the significance threshold, and the enrichment analysis result maps were presented by the "ggplot2" and "GOplot" R packages.

\section{Protein-protein interaction (PPI) network construction and hub DE-MRG alteration analysis}

The Search Tool for the Retrieval of Interacting Genes/Proteins (STRING, https://string-db.org/) database comprises

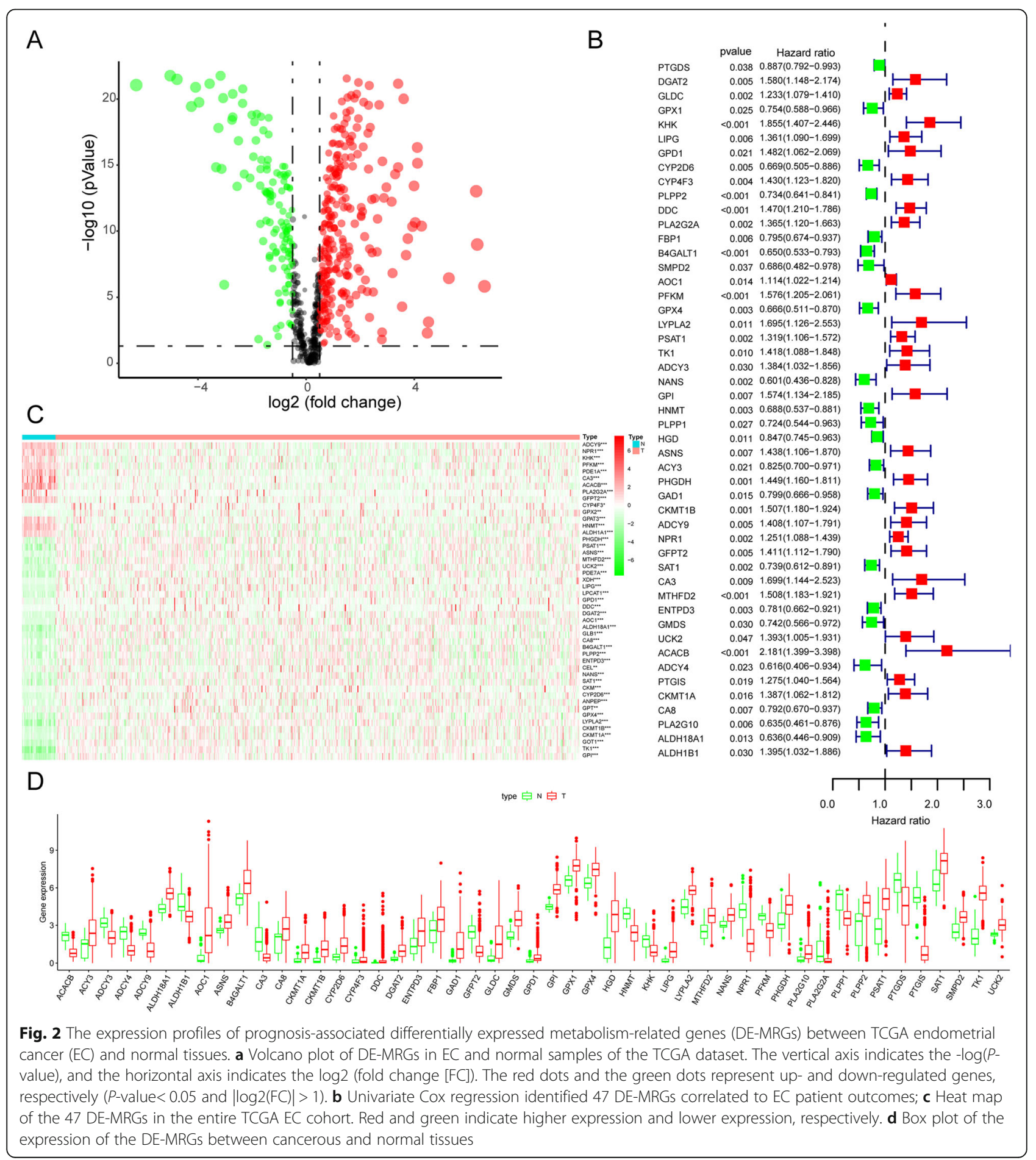


the interaction information of given proteins [14]. Based on the minimum required interaction score setting of 0.4 , we utilized the STRING database to construct a PPI network, which reflected the interactions among the DE-MRG proteins. The network was visualized by Cytoscape software and the top 15 hub proteins were selected based on their connectivity degree in the PPI network [15]. In addition, the alteration landscapes of the hub DE-MRGs in EC were visualized by cBioPortal.

\section{Establishment of a prognostic model based on the DE- MRGs}

We randomly classified all TCGA EC patients into training and testing cohorts. The least absolute shrinkage and selection operator (LASSO) and multivariate Cox regression analysis were then performed to select key prognosis-related DE-MRGs via the "glmnet" $R$ package. The formula of the risk score for the prediction of EC patients' prognosis was as follows: risk score $=$ the sum of the multivariate Cox regression coefficient ratio of each mRNA multiplied by the expression level of each mRNA. Based on the median risk score, we divided the training cohort patients into high- and low-risk subgroups. In the two subgroups, each patient's survival status, OS time, and gene expression profile were presented via the "pheatmap" and "survival" $\mathrm{R}$ packages. In addition, the Kaplan-Meier curve analysis was performed, and receiver operating characteristic (ROC) curves were drawn to estimate the sensitivity and specificity of the prognostic signature.

\section{Validation of the efficacy of the prognostic DE-MRG signature}

The prognostic signature was then introduced into the testing cohort and the entire cohort. Based on the median risk score from the training cohort, the patients in the testing cohort and entire cohort were separated into high- or low-risk groups. Kaplan-Meier curve analysis, time-dependent ROC analysis, and patient outcome distribution were performed.

RNA extraction of clinical samples and quantitative realtime RT-PCR (qRT-PCR) analysis

A total of 30 RNA later-reserved EC specimens were collected from patients who underwent surgery at Jiangsu Province Women and Children Health Hospital (Nanjing, China) between September 2017 and September 2019. All samples were immediately snap frozen in liquid nitrogen and stored at $-80{ }^{\circ} \mathrm{C}$ until further analysis. Total RNA was isolated from fresh-frozen tissues using TRIzol reagent (Invitrogen) from fresh-frozen tissues and transcribed into cDNA using a TaqMan Reverse Transcription kit (Applied Biosystems) with random hexamer primers. qRT-PCR was performed using $2 \times$ SYBR Green qPCR Master Mix (Selleck, Shanghai, China). The housekeeping gene GAPDH was used for normalization of the qRT-PCR data before calculation using the $\Delta \Delta \mathrm{Ct}$ method and Student's t-test (two-tailed) was used for the comparison analyses. The primers used are listed in Supplementary Table 2.

\section{Evaluation of clinical independence and construction of the nomogram}

Next, we removed EC patients who lacked detailed clinicopathological information including survival status and time, age, weight, clinical stage, tumor grade, and lymph node status. The clinicopathological characteristics and the MRG expression data of the remaining patients were compared between the high- and low-risk subgroups and comprehensively displayed in the heatmap. Moreover, the clinical indexes and risk scores were included in univariate and multivariate Cox regression analyses to validate the independence of the risk model. ROC curves for the signature and other clinical features were used to assess the predictive efficacy of the model. In addition, the correlation between the MRGs from the risk model and the clinical index was also measured. Finally, we utilized the "rms" $\mathrm{R}$ package to consolidate the risk score and clinical characteristics for nomogram construction.

Table 1 Univariate Cox regression identified 20 MR-DEGs correlated to endometrial cancer patient OS

\begin{tabular}{|c|c|c|c|c|}
\hline Gene ID & $\mathrm{HR}$ & HR.95 L & $\mathrm{HR} .95 \mathrm{H}$ & $\boldsymbol{P}$-value \\
\hline CYP4F3 & 1.19013465 & 1.08676961 & 1.303330965 & 0.000173367 \\
\hline PHGDH & 1.02042871 & 1.009021493 & 1.031964888 & 0.000422248 \\
\hline GPX2 & 1.002979902 & 1.001288036 & 1.004674627 & 0.000551669 \\
\hline CKMT1B & 1.223457962 & 1.083264863 & 1.381794458 & 0.001162169 \\
\hline CEL & 1.008855015 & 1.003431253 & 1.014308094 & 0.001348837 \\
\hline DDC & 1.098684916 & 1.0372027 & 1.16381161 & 0.001359205 \\
\hline KHK & 1.189854228 & 1.067798895 & 1.325861162 & 0.001644451 \\
\hline YPLA2 & 1.019470527 & 1.006509657 & 1.032598294 & 0.003137784 \\
\hline ACACB & 1.834694401 & 1.218329674 & 2.762883985 & 0.003667941 \\
\hline ASNS & 1.052124561 & 1.015433759 & 1.090141116 & 0.00502135 \\
\hline GPAT3 & 1.104677229 & 1.028736495 & 1.186223863 & 0.006151058 \\
\hline CKMT1A & 1.256355384 & 1.059829197 & 1.489323803 & 0.008551614 \\
\hline CKM & 1.141628713 & 1.031351441 & 1.263697383 & 0.01060172 \\
\hline UCK2 & 1.035172213 & 1.007976028 & 1.063102178 & 0.010933586 \\
\hline HNMT & 0.861666359 & 0.760829865 & 0.975867206 & 0.019044088 \\
\hline SAT1 & 0.997992548 & 0.996252269 & 0.999735867 & 0.024031622 \\
\hline B4GALT1 & 0.995107725 & 0.990877644 & 0.999355864 & 0.024044249 \\
\hline GLB1 & 0.971544079 & 0.946835378 & 0.996897579 & 0.028065649 \\
\hline PSAT1 & 1.007191289 & 1.000533596 & 1.013893283 & 0.034207771 \\
\hline PFKM & 1.087927137 & 1.004895375 & 1.177819587 & 0.037477496 \\
\hline
\end{tabular}

Abbreviations: MR-DEGs metabolism-related differentially expressed genes, OS overall survival, $H R$ Hazard ratio 


\section{Results}

Identification of prognosis-related DE-MRGs

The detailed flow chart for the prognostic predictive model construction in this study is shown in Fig. 1. From the metabolic pathways in the KEGG database, we extracted a total of 944 metabolism-related genes. After the integration of the MRG expression data of 552 TCGA EC cancerous and 35 nontumor samples, we obtained 156 upregulated and 64 downregulated MRGs (Fig. 2a). Univariate Cox regression analysis identified 47 genes significantly correlated with EC patients' OS (Fig. $2 \mathrm{~b}$ and Table 1). The expression pattern of 47 prognosis-related MRGs is shown in the heatmap and box plot in Fig. 2c-d.

Functional enrichment of the prognosis-related DE-MRGs Function annotation analyses of the 220 DE-MRGs and 47 prognostic DE-MRGs were then performed (Supplementary Fig. 1 and Fig. 3). GO enrichment showed that the prognostic MRGs were mainly involved in "carboxylic acid biosynthetic process ", "cofactor binding ", "alpha-amino acid metabolic process " and "cellular amino acid metabolic process" (Fig. 3a). The enriched GO terms and related gene expression profiles are presented in Fig. 3b. KEGG pathway enrichment analysis showed that the prognostic DE-MRGs were mainly involved in "purine metabolism", "biosynthesis of amino acids", "glycolysis / gluconeogenesis" and "glycerophospholipid metabolism" (Fig. 4a). The expression levels of the correlated genes in the enriched KEGG pathways are displayed in the heatmap (Fig. 4b).

\section{The PPI network of 47 prognosis-related DE-MRGs and hub gene alteration analysis}

Through the STRING website, we built a PPI network of the 47 DE-MRG proteins (Supplementary Fig. 2). There were 47 nodes and 81 edges included in the network based on the interaction score criteria. The top 15 hub proteins with the highest connectivity degrees in the PPI network were as follows: ALDH18A1, GOT1, DGAT2, GPD1, GPI, PHGDH, GPT, ENTPD3, ASNS, ADCY9, CKMT1A, LPCAT1, MTHFD2, PPAP2C and PSAT1

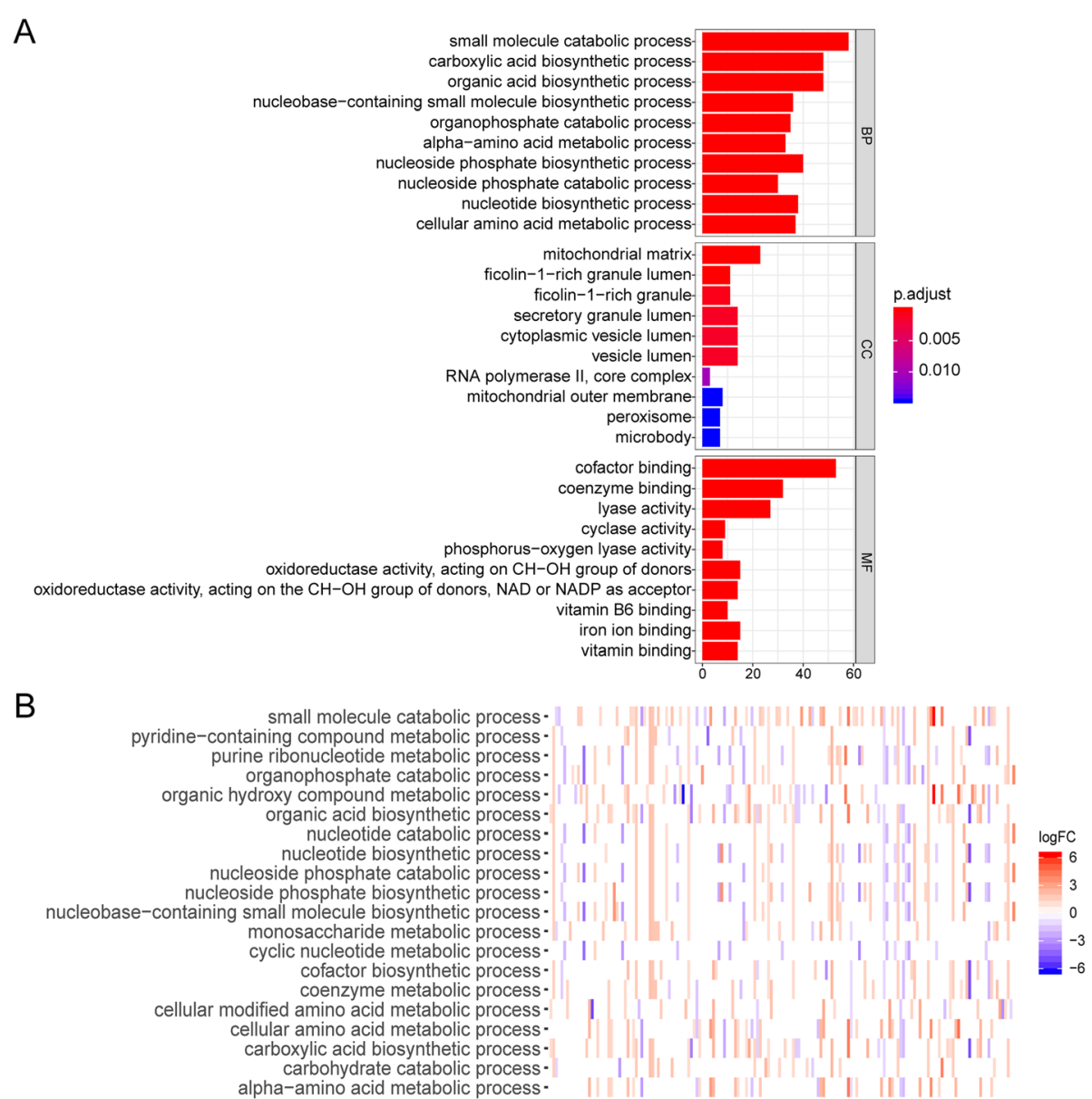

Fig. 3 Gene Ontology (GO) functional enrichment of prognostic DE-MRGs. a GO analysis shows the biological processes, cellular component, and molecular functions involved in differential genes. $\mathbf{b}$ Heatmap of the expression of DEGs in the enriched GO items 


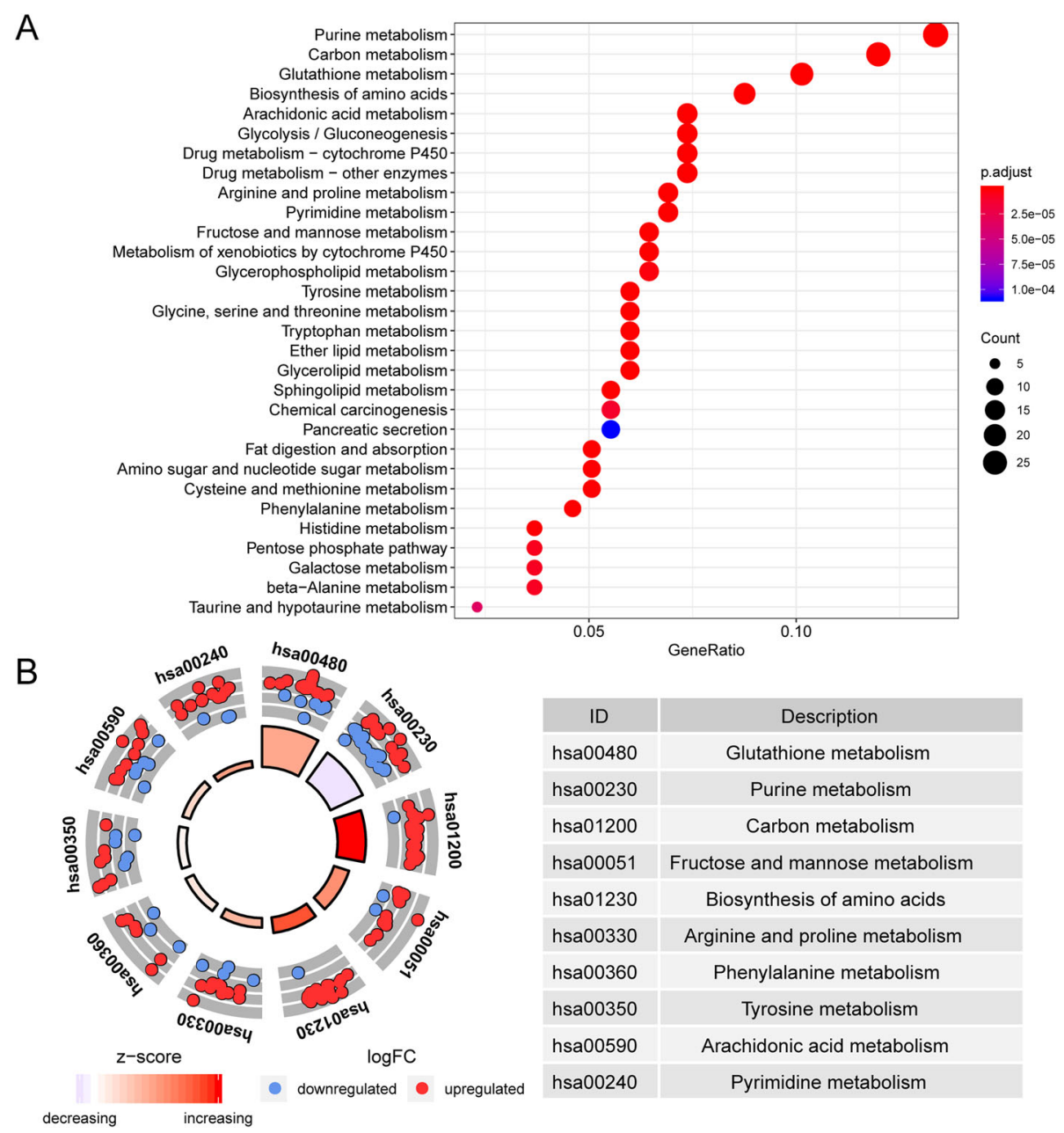

Fig. 4 Kyoto Encyclopedia of Genes and Genomes (KEGG) pathways enrichment of prognosis-associated DE-MRGs. a KEGG analysis shows significantly enriched pathways of DE-MRGs. The node color changes gradually from red to blue in descending order according to the adjusted $P$-values. The size of the node represents the number of counts. $\mathbf{b}$ Circle plot of the enriched DEGs in the KEGG items

(Fig. 5a-b). The alteration results of the hub genes showed that GPI, GPT, ADCY9, and LPCAT1 ranked as the most frequently altered genes. GPI, GPT, and LPCA $\mathrm{T} 1$ were frequently overamplified in endometrial cancer patients, while ADCY9 more often had missense mutations (unknown significance) (Fig. 5c).

\section{Identification of a nine DE-MRG-based prognostic model} Next, we randomly divided the 541 TCGA EC patients into a training cohort $(n=272)$ and a testing cohort $(n=269)$. LASSO and multivariate Cox regression analyses identified 9 genes significantly associated with prognosis: CYP4F3, CEL, GPAT3, LYPLA2, HNMT, PHGDH, CKM, UCK2 and ACACB (Fig. 6 and Table 2). According to the results of multivariate Cox regression analysis, we constructed the prognostic model as follows: risk score $=(0.110103 \times$ expression value of CYP4F3 $)+$ $(0.013456 \times$ expression value of $\mathrm{CEL})+(0.104444 \times$ expression value of GPAT3 $)+(0.017777 \times$ expression value of LYPLA2 $)+(-0.10986 \times$ expression value of $\mathrm{HNMT})+(0.017183 \times$ expression value of $\mathrm{PHGDH})+$ $(0.200964 \times$ expression value of CKM $)+(0.051913 \times$ expression value of $\mathrm{UCK} 2)+(0.634313 \times$ expression value of ACACB).

Based on the mean risk score from the training cohort, the patients were divided into high-risk $(n=136)$ and low-risk $(n=136)$ subgroups. Each individual's risk score and survival status were ranked and displayed on the dot plot, which showed significant differences in OS between the groups (Fig. 7a-b). Likewise, the Kaplan-Meier curve analysis demonstrated that the OS of the higher-risk group was significantly shorter than that of the low-risk group $(P=1.971 \mathrm{e}-06)$ (Fig. $7 \mathrm{~d})$. The expression profiles of the 9 prognostic DE-MRGs showed that UCK2, PHGDH, ACACB, LYPLA2, CYP4F3, GPAT3, CEL, and CKM were expressed at higher levels in the high-risk 


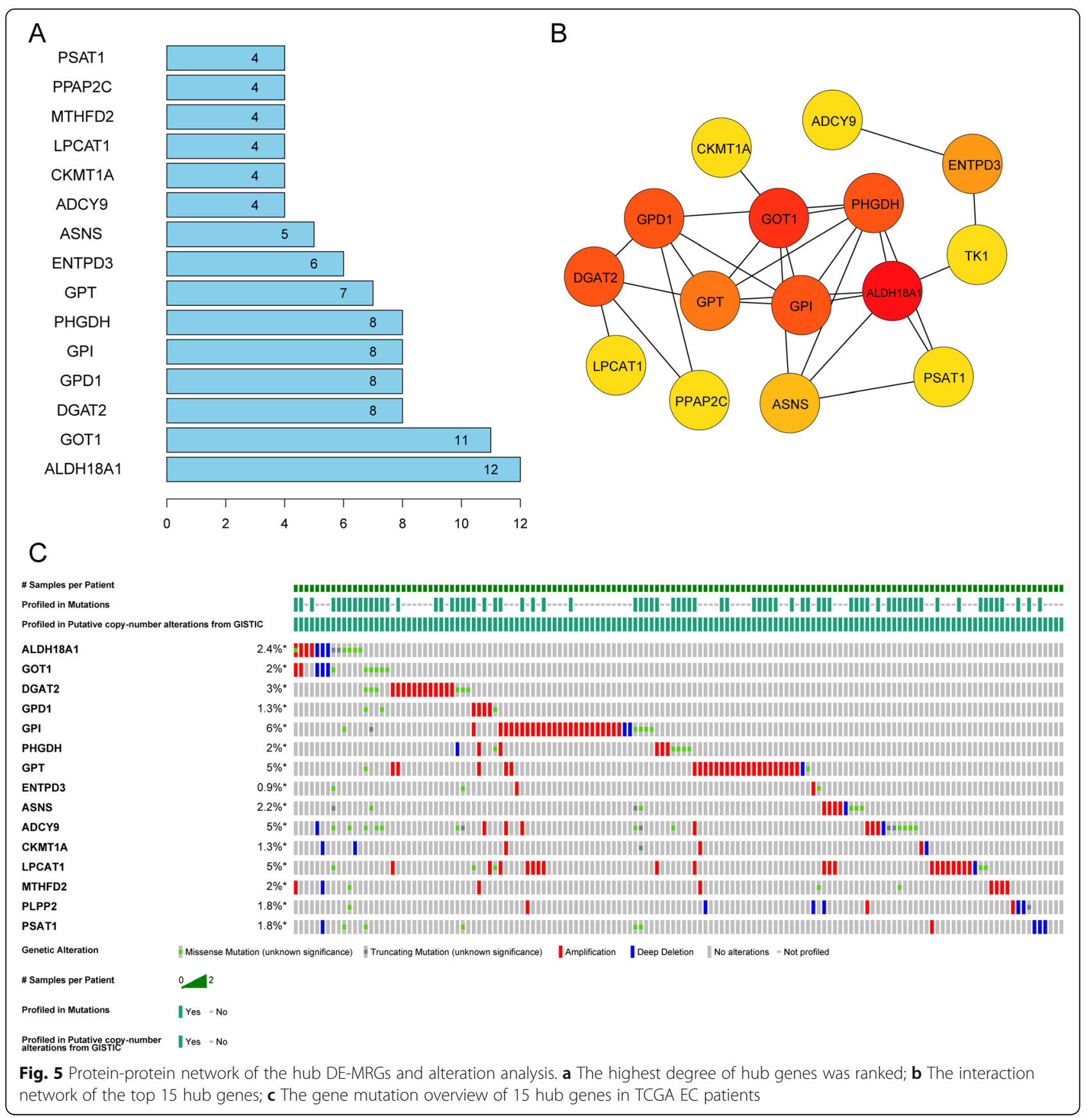

subgroup, while HNMT was expressed at lower levels (Fig. 7c). ROC curve analysis revealed that the area under the ROC curve (AUC) of the prognostic MRG model was 0.771 (Fig. 7e).

\section{Validation of the efficacy of the 9-MRG prognostic signature}

The risk model was then introduced into the testing cohort and entire cohort, and each individual's risk score was calculated. Based on the training cohort cut-off risk score, the patients in the testing cohort were classified as
121 high-risk and 148 low-risk individuals. In agreement with the results from the training cohort, the survival status (Fig. 8a), survival time (Fig. 8b) and KM curve analysis of the high-risk subgroup presented worse outcomes than those of the low-risk subgroup in the testing cohort with a shorter overall survival time $(P=4.151$ e04) (Fig. 8d). The expression patterns of the 9 MRGs were consistent with those in the training cohort (Fig. $8 \mathrm{c})$, and the AUC of the risk model in the testing cohort was 0.796 (Fig. 8e). Similar results were also observed for the entire cohort. The low-risk subgroup presented 

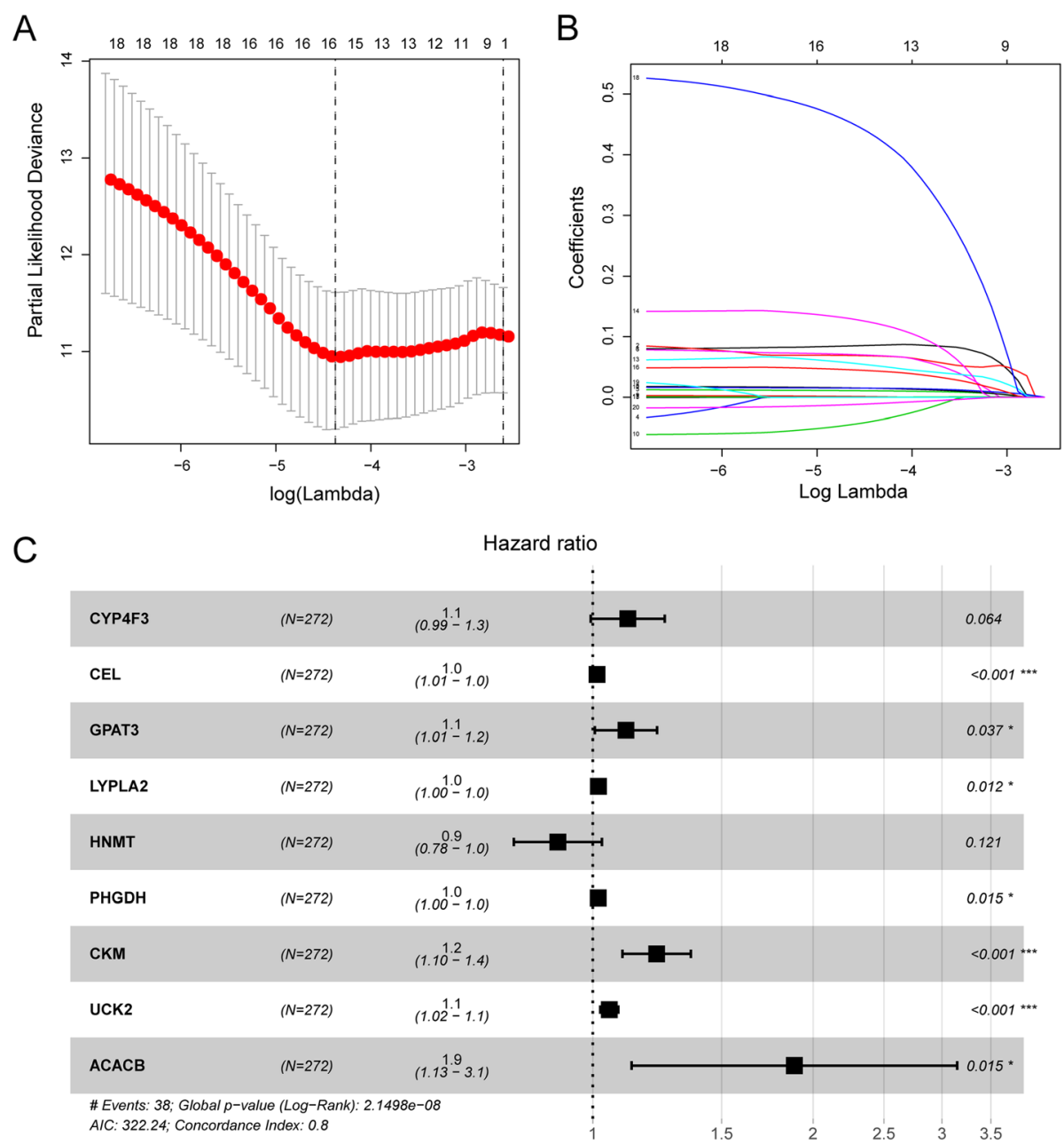

Fig. 6 Subsequent identification of prognosis related DE-MRGs using LASSO and Cox regression analysis. a Plots of the cross-validation error rates. Each dot represents a lambda value along with error bars to give a confidence interval for the cross-validated error rate; $\mathbf{b}$ LASSO coefficient profiles of the MRGs associated with the overall survival of endometrial cancer; $\mathbf{c}$ multivariate Cox regression identified 9 prognostic MRGs in the training cohort

Table 2 Multivariate Cox regression selected 9 MR-DEGs correlated to endometrial cancer patient OS

\begin{tabular}{lllll}
\hline Gene ID & HR & HR.95 L & HR.95H & $\boldsymbol{P}$-value \\
\hline CYP4F3 & 1.116392881 & 0.99351779 & 1.25446477 & 0.064219393 \\
CEL & 1.013547388 & 1.007787405 & 1.019340292 & $3.69768 \mathrm{E}-06$ \\
GPAT3 & 1.110093426 & 1.006471947 & 1.224383272 & 0.03670839 \\
LYPLA2 & 1.017935764 & 1.003942684 & 1.03212388 & 0.011831278 \\
HNMT & 0.895961269 & 0.77990526 & 1.029287321 & 0.1206327 \\
PHGDH & 1.017331051 & 1.003306749 & 1.031551385 & 0.015262521 \\
CKM & 1.222580744 & 1.097931508 & 1.36138153 & 0.00024948 \\
UCK2 & 1.053283808 & 1.024064937 & 1.083336359 & 0.000298414 \\
ACACB & 1.885726048 & 1.129790043 & 3.147454476 & 0.015230463 \\
\hline Ab
\end{tabular}

Abbreviations: MR-DEGs metabolism-related differentially expressed genes, OS overall survival, $H R$ Hazard ratio longer survival times and better survival statuses (Fig. 9a-b). In addition, the expression profiles of the 9 MRGs were in line with those in both the training cohort and the testing cohort (Fig. 9c). Kaplan-Meier curve analysis showed that the low-risk subgroup had longer OS times $(P=1.242 \mathrm{e}-08)$ (Fig. 9d), and ROC curve analysis showed that the AUC of the model was 0.78 (Fig. 9e).

\section{Validation of the expression levels of the 9 MRGs in clinical samples}

The expression signatures of the 9 MRGs were subsequently explored in 30 endometrial cancer clinical specimens. The results demonstrated that UCK2, PHGDH, ACACB, LYPLA2, CYP4F3, GPAT3, CEL, and CKM mRNA level were upregulated in cancerous tissues, while HNMT was downregulated, which was in accordance with the above findings (Fig. 10a-i). 
A

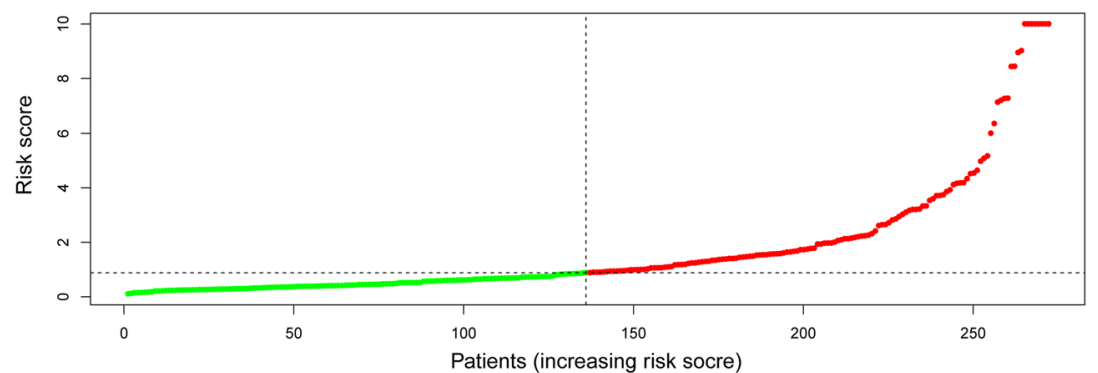

B

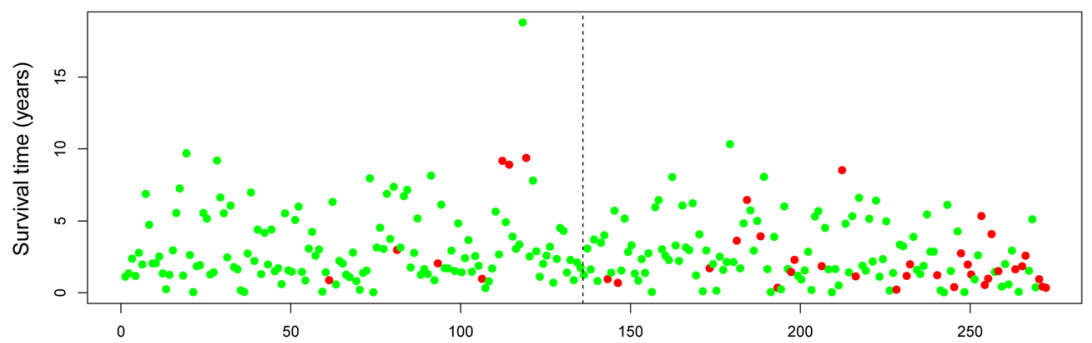

C

Patients (increasing risk socre)

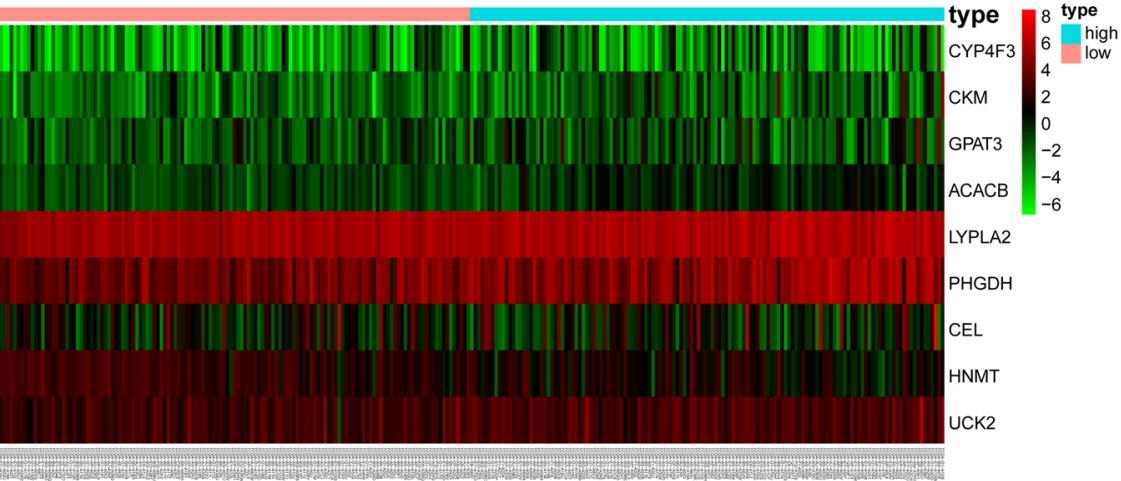

D

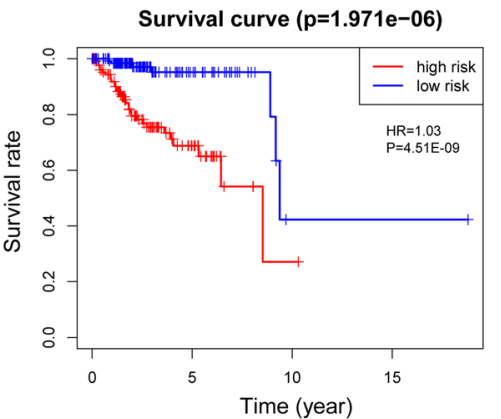

$E$

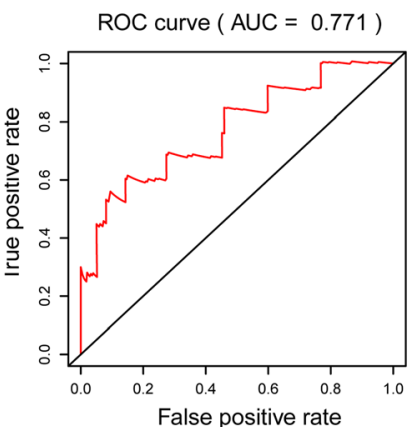

Fig. 7 Prognostic analysis of the model in the TCGA training cohort. a The risk score, (b) survival status, (c) expression heatmap, (d) Kaplan-Meier survival, and (e) time-dependent ROC curves of the prognostic model for the TCGA EC training cohort

The clinical independence and correlation estimation of the risk signature

Then, we combined the risk model with other clinical factors and performed univariate and multivariate analyses to examine the clinical independence of the model. The results showed that the model was able to serve as an independent prognostic indicator (both $P<0.001$ ) (Fig. 11a-b). The AUC value of the prognostic model was 0.781 , which was significantly higher than that of patients' age $(\mathrm{AUC}=0.535)$ and weight $(\mathrm{AUC}=0.633)$, clinical-stage $(A U C=0.710)$, tumor grade $(A U C=0.656)$, histology (AUC $=0.522$ ), and lymph node status (AUC = 0.697) (Fig. 11c). Next, we assessed the risk scores, clinical features, and nine-gene expression profiles of EC patients and displayed them in the heatmap shown in Fig. 11d. Interestingly, the clinical characteristics of the patients were highly in accordance with the risk level calculated from the model. The high-risk subgroup 
A

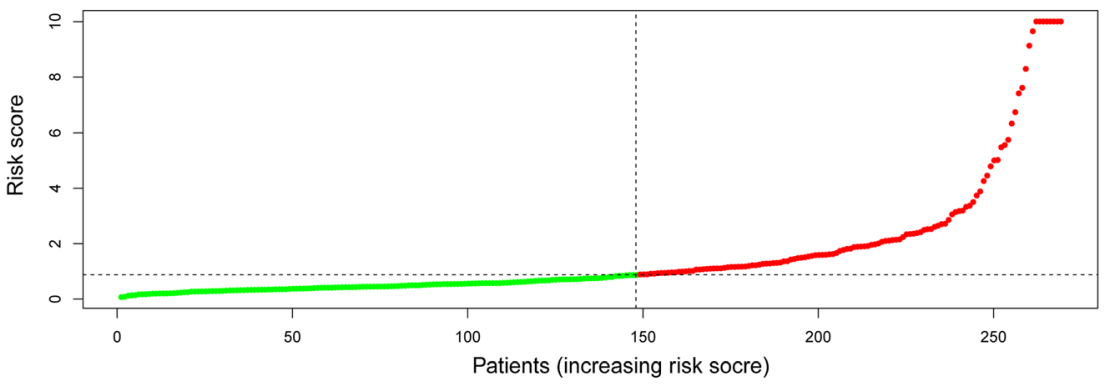

B

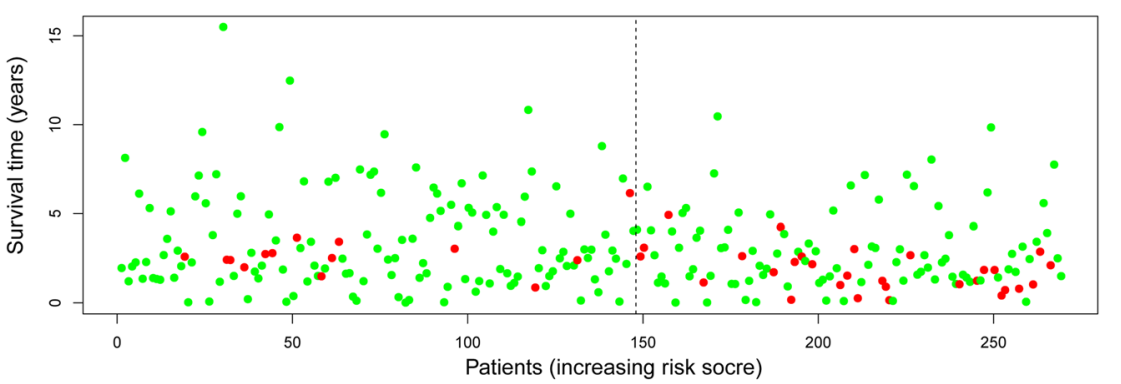

C

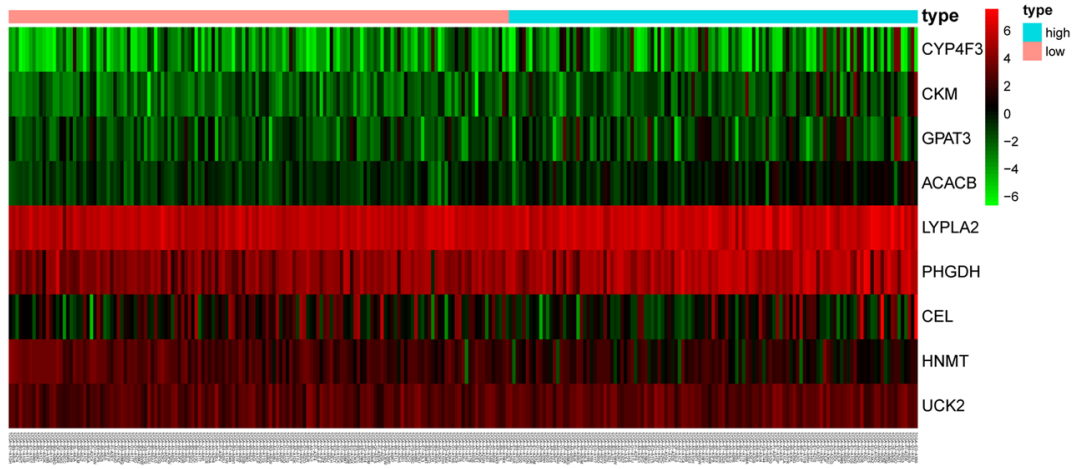

D

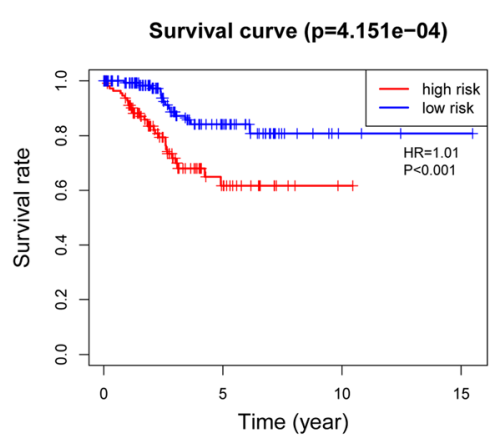

$\mathrm{E}$

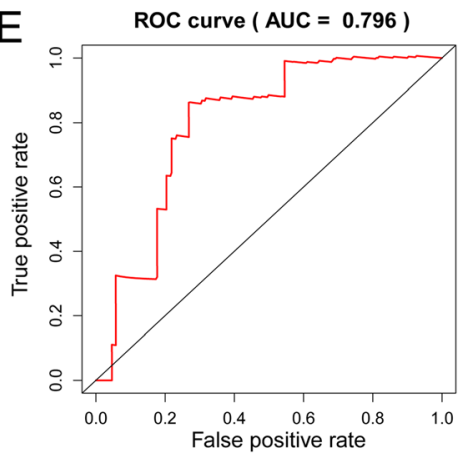

Fig. 8 Validation of the efficacy of the risk signature in the TCGA testing cohort. a The risk score, (b) survival status, (c) expression heatmap, (d) Kaplan-Meier survival, and (e) time-dependent ROC curves of the prognostic model for the TCGA EC testing cohort

patients were characterized by late-stage, high-grade, serous carcinoma, and more metastatic lymph nodes (Fig. 11d-e), which all presented worse outcomes. The correlations between each gene from the prognostic model and the patients' clinical features were also measured. PHGDH, ACACB, HNMT, CYP4F3, and LYPLA2 were shown to be significantly associated with patient prognosis (Fig. 11f). The other clinical features of each prognostic MRG from the signature are presented in Supplementary Fig. 3.

\section{Nomogram building and validation}

Based on the patients' risk scores and clinical features, we built a comprehensive prognostic nomogram to estimate EC patients' survival probability for 5 years based on the entire TCGA set. Seven independent prognostic 

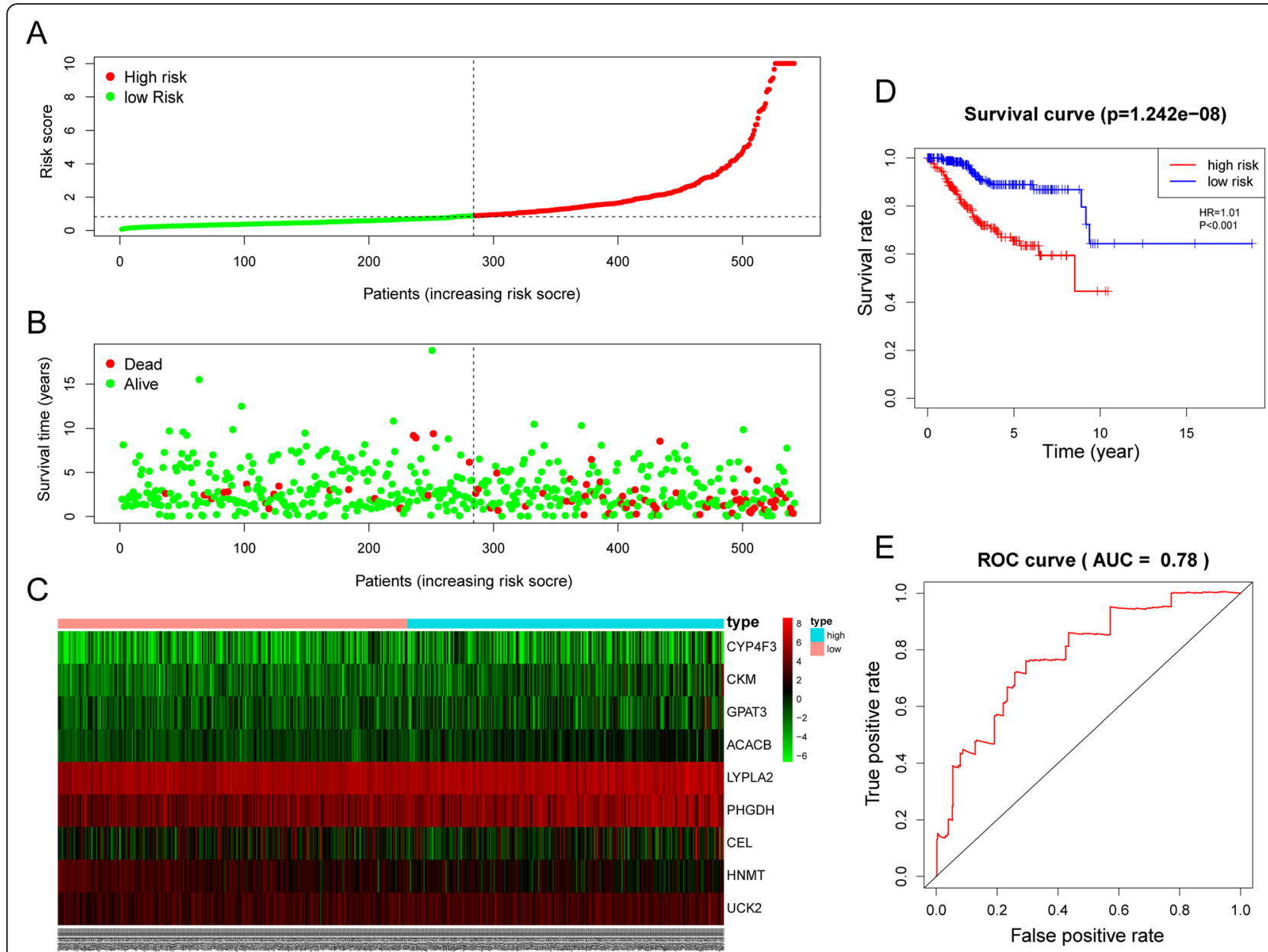

Fig. 9 Estimation of the efficacy of the risk model in TCGA entire EC cohort. a The risk score, (b) survival status, (c) expression heatmap, (d) Kaplan-Meier survival, and (e) time-dependent ROC curves of the prognostic model for the TCGA EC entire cohort

parameters, including metabolic risk signature, age, grade, weight, histology, stage and lymph node status, were integrated into the nomogram (Fig. 12a). The calibration plots showed excellent consistency between the nomogram predictions and actual observations in terms of the 3- and 5-year survival rates in the TCGA cohort (Fig. 12b-c).

\section{Discussion}

Metabolic abnormalities have recently been widely studied and shown to play an important role in tumor development in various cancer types. Metabolic dysfunction in the tumor microenvironment could lead to various outcomes in patients, and metabolism-related genes can be used as prognostic markers of tumors. In this work, we thoroughly investigated the implications of metabolism-related genes in endometrial cancer progression. By analyzing the mRNA data of TCGA EC patients, we obtained 220 dysregulated MRGs, among which 47 were associated with EC patients' OS. Functional enrichment analysis of these prognostic MRGs showed that they were closely associated with the cellular amino acid metabolic process, glycolysis, and glycerophospholipid metabolism. In accordance with our observation, Byrne et al. also found that glycolysis and lipogenesis are highly associated with endometrial cancer phenotypes and that the suppression of GLUT6 gene expression could inhibit glycolysis and the survival of EC cells, underlying the crucial role of energy metabolism in tumor progression [16]. In addition, our results further revealed the exact dysregulated metabolic genes of these disordered metabolism-related pathways, which may provide a new perspective on the molecular mechanisms of metabolism alterations in tumor progression.

Metabolic prognostic risk signatures that combined the expression of multiple metabolism-related genes have been indicated to serve as powerful prognostic indicators in various malignant diseases, such as glioma, liver cancer, ovarian cancer, and papillary thyroid carcinoma. Zhou et al. identified a 29-energy metabolism- 

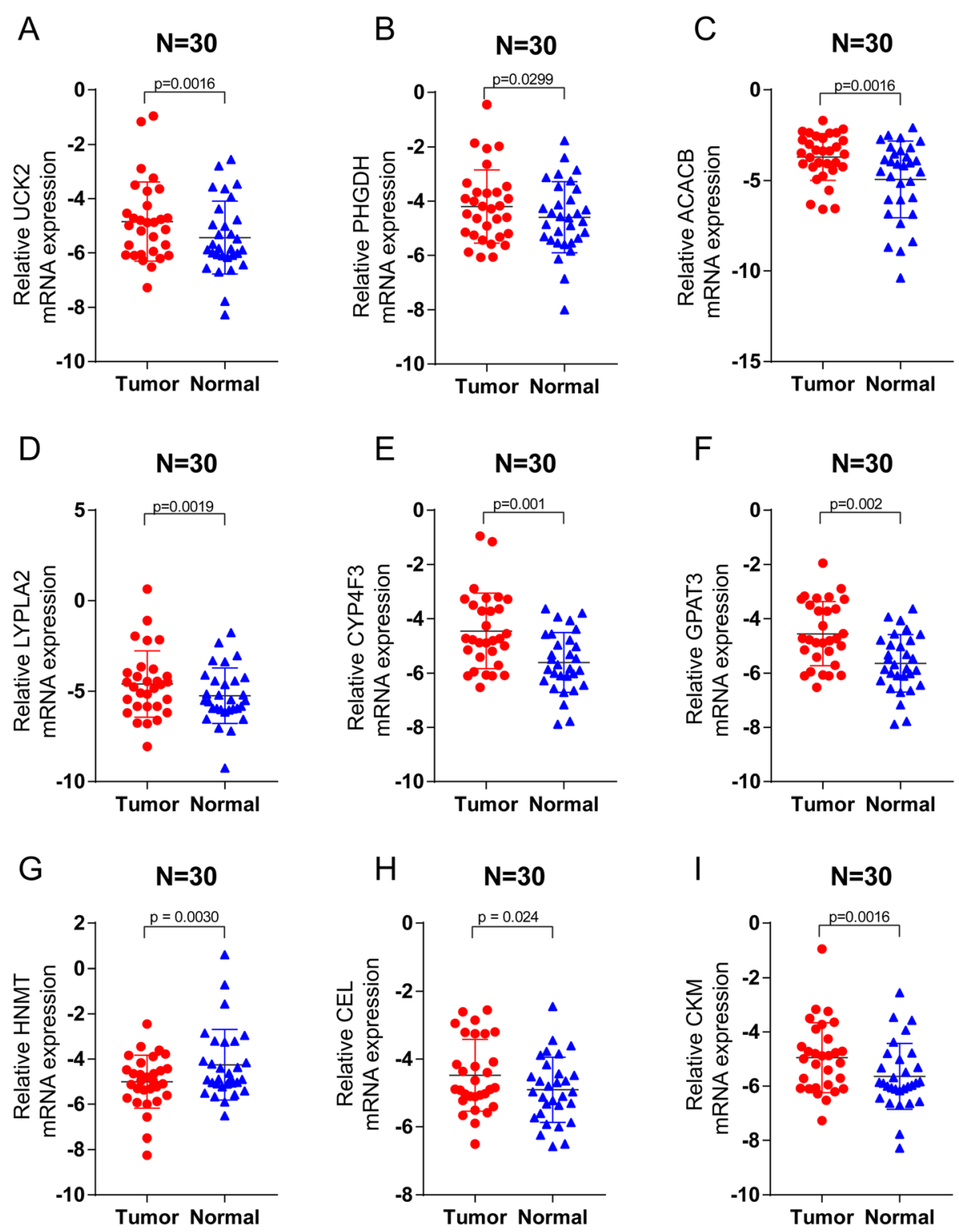

Fig. 10 Validation of the expression signature of 9 MRGs in tissues by qRT-PCR. Student's t-test (two-tailed) was used for the comparison analyses and the significance threshold was set at 0.05 for in each test

related gene signature, containing branched-chain amino acid transaminase 1 (BCAT1), interleukin-4 and carbohydrate sulfotransferases, to evaluate the prognosis of diffuse glioma [17]. Wang et al. enrolled 6 risks and 2 protective metabolic genes into the prognostic metabolic model which effectively predicted ovarian cancer patients' prognosis [18]. Likewise, Ma et al. developed a metabolic gene signature as a biomarker for dedifferentiated thyroid cancer [19], and Liu et al. built a fourmetabolic gene signature for liver cancer patient outcome prediction [20].

In the present study, we performed LASSO and multivariate Cox regression analyses and identified a nine- gene signature including CYP4F3, CEL, GPAT3, LYPL A2, HNMT, PHGDH, CKM, UCK2, and ACACB. Among them, HNMT was considered a protective factor while others were risk factors. The diagnostic and predictive effectiveness of these prognostic genes has already been reported in other studies. Cui et al. reported a significantly higher expression of carboxyl ester lipase (CEL) in breast cancer. The combination of CEL and other biomarkers could improve the diagnostic capability for breast cancer [21]. Likewise, Richard et al. found that over $70 \%$ of estrogen receptor (ER)-negative breast cancers exhibited elevated phosphoglycerate dehydrogenase (PHGDH) protein expression, which is 


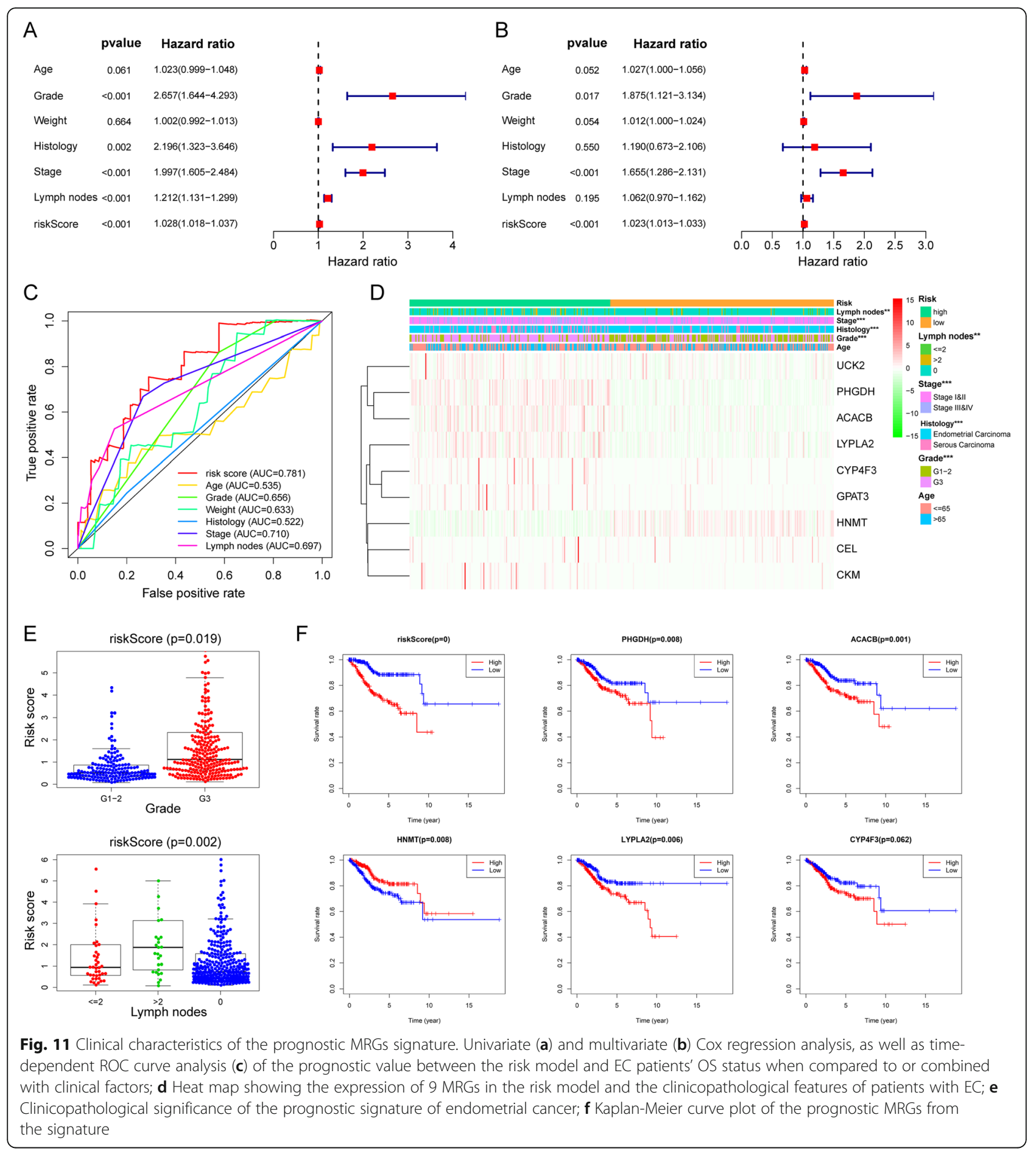

crucial for promoting serine pathway flux [22]. Li reported downregulation of PHGDH caused by overexpressing LncRNA PlncRNA-1 mediated cell apoptosis rate in breast cancer [23]. In addition, Zhang et al. discovered that PHGDH could define a metabolic subtype in lung adenocarcinomas with unique metabolic dependencies [24]. In pancreatic ductal adenocarcinoma, CYP4F3, one isoform of the cytochrome P450 (CYP) superfamily, was shown to be upregulated in tumor tissues and could serve as a distinguishing marker [25]. Uridine-cytidine kinase 2(UCK2) was positively correlated with early recurrence and poor prognosis in hepatocellular carcinoma. Overexpression of UCK2 increased MMP2/9 expression and further activated Stat3 signaling, mediating the metastasis of hepatocellular carcinoma cells [26]. For ACACB, Lally et al. showed that 


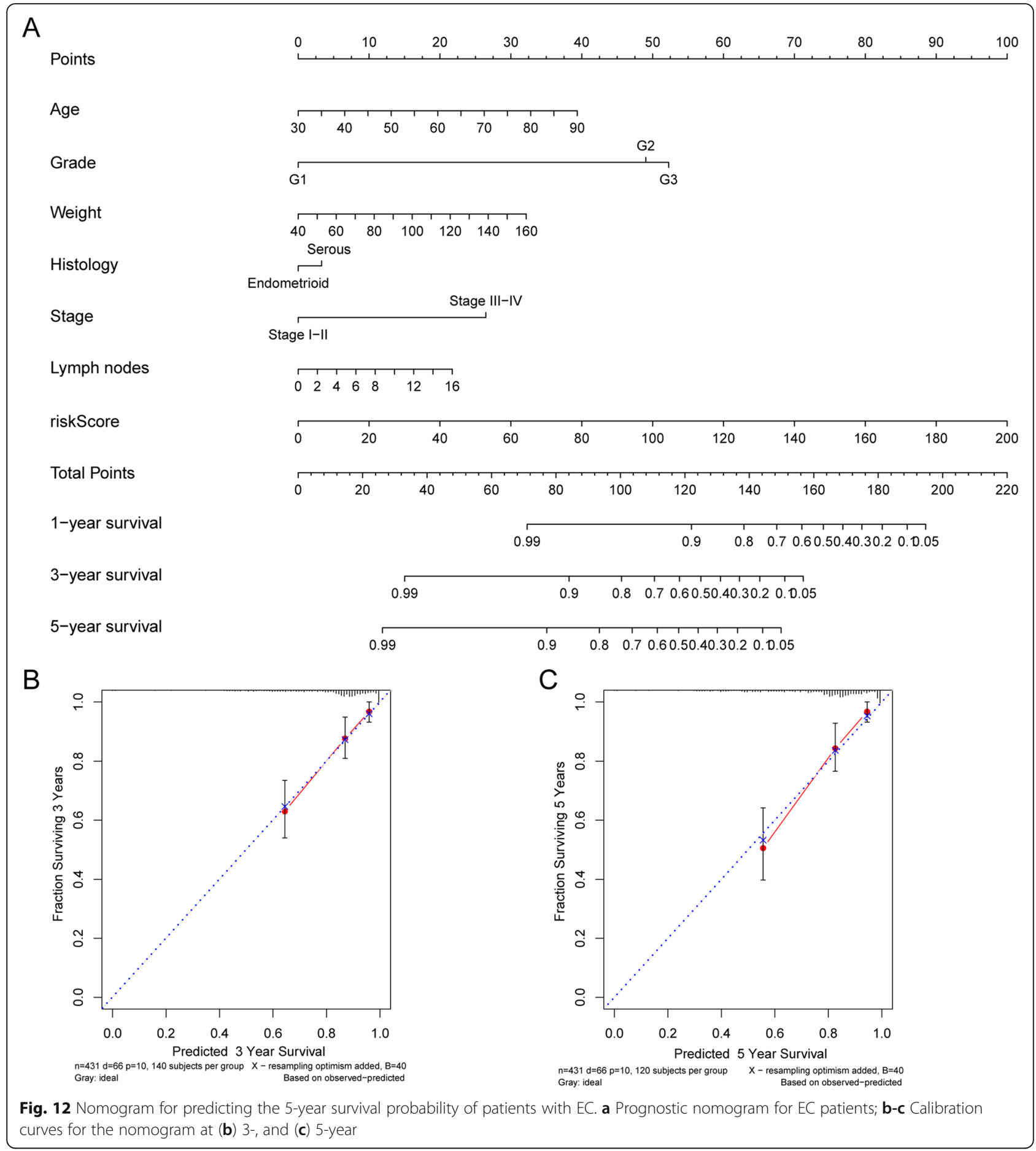

humans with fatal HCC subtypes have increased acetyl-CoA carboxylase (ACC) expression and that the genetic activation of ACC promoted the formation of hepatic de novo lipids and induced subsequent liver carcinogenesis [27].

Here, through bioinformatic analysis and outside validation, we innovatively reported that these metabolic genes are closely related to the prognosis of EC patients.
In addition, the metabolic risk signature combining these genes could accurately categorize EC patients into high- or low-risk subgroups which represented patients' long-term outcomes. Last, our study was the first to build a comprehensive nomogram that incorporated a metabolism-related signature with clinical features including age, stage, tumor grade and lymph node status to effectively predict the survival of EC patients. This 
prognostic scoring system could provide a precise method to help both physicians and patients perform individualized survival evaluations and select treatment options.

\section{Conclusion}

In conclusion, we identified 47 prognosis-related dysregulated metabolic genes in EC. The prognostic DE-MRGs were highly associated with amino acid, glycolysis, and glycerophospholipid metabolism. The top 15 hub genes in the PPI network were also identified and analyzed. We performed LASSO and multivariate Cox regression analyses to establish and validate a robust prognostic risk signature enrolling the nine dysregulated MRGs. In addition, a comprehensive nomogram that combined clinical characteristics and the risk model was constructed, and its efficacy in predicting EC patients' prognosis was also demonstrated. The 9-MRG model and nomogram may guide the selection of rational therapeutic strategies for doctors in clinical practice.

\section{Supplementary information}

Supplementary information accompanies this paper at https://doi.org/10. 1186/s12885-020-07345-8.

\section{Additional file 1: Supplementary Table 1. The gene list of all metabolic genes.}

Additional file 2: Supplementary Table 2. QRT-PCR Primers for nine metabolic genes

Additional file 3: Supplementary Fig. 1. GO and KEGG pathway enrichment of 220 DE-MRGs.

Additional file 4: Supplementary Fig. 2. The protein-protein interaction network of 47 DE-MRGs.

Additional file 5: Supplementary Fig. 3. Clinical characteristics of each prognostic MRG from the signature.

\section{Abbreviations}

EC: Endometrial cancer; MRGs: Metabolism-related genes; TCGA: The Cancer Genome Atlas; GO: Gene ontology; KEGG: Kyoto Encyclopedia of Genes and Genomes; PPI: Protein-protein interaction; STRING: Search Tool for the Retrieval of Interacting Genes Database; ROC: Receiver operating characteristic; AUC: Area under the curve; LASSO: Least Absolute Shrinkage and Selection Operator; OS: Overall survival; FC: Fold change

\section{Acknowledgements}

Not applicable.

Fundings

Not applicable.

\section{Authors' contributions}

SLF and PPJ designed the project. WS and NMS contributed to data analysis and prepared the main manuscript. XHH and PPJ revised and submitted the manuscript. All authors read and approved the final manuscript.

\section{Availability of data and materials}

The expression data were deposited in the TCGA database and the clinical information was retrieved from the cBioPortal website. Besides, please contact the author for data and materials requests.

\section{Ethics approval and consent to participate}

Ethical consent was approved by the Committees for Ethical Review of Research involving Human Subjects at Nanjing Medical University. Written informed consent was obtained from each patient before sample collection.

\section{Consent for publication}

Not applicable.

\section{Competing interests}

The authors declare that they have no competing interests.

Received: 14 June 2020 Accepted: 14 August 2020

Published online: 07 September 2020

References

1. Siegel RL, Miller KD, Jemal A. Cancer statistics, 2019. CA Cancer J Clin. 2019; 69(1):7-34.

2. Koh WJ, Abu-Rustum NR, Bean S, Bradley K, Campos SM, Cho KR, Chon HS, Chu C, Clark R, Cohn D, et al. Cervical Cancer, version 3.2019, NCCN clinical practice guidelines in oncology. J Natl Compr Cancer Netw. 2019;17(1):6484.

3. Brooks RA, Fleming GF, Lastra RR, Lee NK, Moroney JW, Son CH, Tatebe K, Veneris JL. Current recommendations and recent progress in endometrial cancer. CA Cancer J Clin. 2019:69(4):258-79.

4. Warburg O. On the origin of cancer cells. Science (New York, NY). 1956; 123(3191):309-14.

5. Sonveaux P, Vegran F, Schroeder T, Wergin MC, Verrax J, Rabbani ZN, De Saedeleer CJ, Kennedy KM, Diepart C, Jordan BF, et al. Targeting lactatefueled respiration selectively kills hypoxic tumor cells in mice. J Clin Invest. 2008:118(12):3930-42.

6. Whitaker-Menezes D, Martinez-Outschoorn UE, Flomenberg N, Birbe RC, Witkiewicz AK, Howell A, Pavlides S, Tsirigos A, Ertel A, Pestell RG, et al. Hyperactivation of oxidative mitochondrial metabolism in epithelial cancer cells in situ: visualizing the therapeutic effects of metformin in tumor tissue. Cell Cycle. 2011;10(23):4047-64.

7. Sun L, Suo C, Li ST, Zhang H, Gao P. Metabolic reprogramming for cancer cells and their microenvironment: beyond the Warburg effect. Biochim Biophys Acta Rev Cancer. 2018;1870(1):51-66.

8. Vander Heiden MG, Cantley LC, Thompson CB. Understanding the Warburg effect: the metabolic requirements of cell proliferation. Science (New York, NY). 2009;324(5930):1029-33.

9. Pavlova NN, Thompson CB. The emerging hallmarks of Cancer metabolism. Cell Metab. 2016;23(1):27-47.

10. Cerami E, Gao J, Dogrusoz U, Gross BE, Sumer SO, Aksoy BA, Jacobsen A Byrne CJ, Heuer ML, Larsson E, et al. The cBio cancer genomics portal: an open platform for exploring multidimensional cancer genomics data. Cancer Discov. 2012;2(5):401-4.

11. Kanehisa M, Furumichi M, Tanabe M, Sato Y, Morishima K. KEGG: new perspectives on genomes, pathways, diseases and drugs. Nucleic Acids Res. 2017:45(D1):D353-61.

12. Ritchie ME, Phipson B, Wu D, Hu Y, Law CW, Shi W, Smyth GK. limma powers differential expression analyses for RNA-sequencing and microarray studies. Nucleic Acids Res. 2015;43(7):e47.

13. Ashburner M, Ball CA, Blake JA, Botstein D, Butler H, Cherry JM, Davis AP, Dolinski K, Dwight SS, Eppig JT, et al. Gene ontology: tool for the unification of biology. The gene ontology consortium. Nat Genet. 2000;25(1):25-9.

14. Szklarczyk D, Gable AL, Lyon D, Junge A, Wyder S, Huerta-Cepas J, Simonovic M, Doncheva NT, Morris JH, Bork P, et al. STRING v11: proteinprotein association networks with increased coverage, supporting functional discovery in genome-wide experimental datasets. Nucleic Acids Res. 2019; 47(D1):D607-13.

15. Shannon P, Markiel A, Ozier O, Baliga NS, Wang JT, Ramage D, Amin N, Schwikowski B, Ideker T. Cytoscape: a software environment for integrated models of biomolecular interaction networks. Genome Res. 2003;13(11): 2498-504.

16. Byrne FL, Poon IK, Modesitt SC, Tomsig JL, Chow JD, Healy ME, Baker WD, Atkins KA, Lancaster JM, Marchion DC, et al. Metabolic vulnerabilities in endometrial cancer. Cancer Res. 2014;74(20):5832-45.

17. Zhou Z, Huang R, Chai R, Zhou X, Hu Z, Wang W, Chen B, Deng L, Liu Y, Wu F. Identification of an energy metabolism-related signature associated with clinical prognosis in diffuse glioma. Aging. 2018;10(11):3185-209. 
18. Wang $L, L i X$. Identification of an energy metabolismrelated gene signature in ovarian cancer prognosis. Oncol Rep. 2020;43(6):1755-70.

19. Ma B, Jiang H, Wen D, Hu J, Han L, Liu W, Xu W, Shi X, Wei W, Liao T, et al. Transcriptome analyses identify a metabolic gene signature indicative of dedifferentiation of papillary thyroid Cancer. J Clin Endocrinol Metab. 2019; 104(9):3713-25.

20. Liu GM, Xie WX, Zhang CY, Xu JW. Identification of a four-gene metabolic signature predicting overall survival for hepatocellular carcinoma. J Cell Physiol. 2020;235(2):1624-36.

21. Cui $Y$, Jiao $Y$, Wang $K$, He M, Yang Z. A new prognostic factor of breast cancer: high carboxyl ester lipase expression related to poor survival. Cancer Gene Ther. 2019;239:54-61.

22. Possemato R, Marks KM, Shaul YD, Pacold ME, Kim D, Birsoy K, Sethumadhavan S, Woo HK, Jang HG, Jha AK, et al. Functional genomics reveal that the serine synthesis pathway is essential in breast cancer. Nature. 2011:476(7360):346-50.

23. Li Q, Gao H, Zhou S, Liao Y. LncRNA PIncRNA-1 overexpression inhibits the growth of breast cancer by upregulating TGF- $\beta 1$ and downregulating PHGD H. Breast Cancer (Tokyo, Japan). 2018;25(5):619-25.

24. Zhang B, Zheng A, Hydbring P, Ambroise G, Ouchida AT, Goiny M, Vakifahmetoglu-Norberg H, Norberg E. PHGDH defines a metabolic subtype in lung adenocarcinomas with poor prognosis. Cell Rep. 2017;19(11):2289303 .

25. Gandhi AV, Saxena S, Relles D, Sarosiek K, Kang CY, Chipitsyna G, Sendecki $J A$, Yeo CJ, Arafat HA. Differential expression of cytochrome P450 omegahydroxylase isoforms and their association with clinicopathological features in pancreatic ductal adenocarcinoma. Ann Surg Oncol. 2013;20(Suppl3): S636-43.

26. Zhou Q, Jiang H, Zhang J, Yu W, Zhou Z, Huang P, Wang J, Xiao Z. Uridinecytidine kinase 2 promotes metastasis of hepatocellular carcinoma cells via the Stat3 pathway. Cancer Manag Res. 2018;10:6339-55.

27. Lally JSV, Ghoshal S, DePeralta DK, Moaven O, Wei L, Masia R, Erstad DJ, Fujiwara N, Leong V, Houde VP, et al. Inhibition of Acetyl-CoA Carboxylase by Phosphorylation or the Inhibitor ND-654 Suppresses Lipogenesis and Hepatocellular Carcinoma. Cell Metab. 2019:29(1):174-182.e175.

\section{Publisher's Note}

Springer Nature remains neutral with regard to jurisdictional claims in published maps and institutional affiliations.

Ready to submit your research? Choose BMC and benefit from:

- fast, convenient online submission

- thorough peer review by experienced researchers in your field

- rapid publication on acceptance

- support for research data, including large and complex data types

- gold Open Access which fosters wider collaboration and increased citations

- maximum visibility for your research: over $100 \mathrm{M}$ website views per year

At $\mathrm{BMC}$, research is always in progress.

Learn more biomedcentral.com/submissions 